Article

\title{
Unit Commitment Accommodating Large Scale Green Power
}

\author{
Yuntao Ju ${ }^{1, *}$, Jiankai Wang ${ }^{1}$, Fuchao Ge ${ }^{2}$, Yi Lin ${ }^{3}$, Mingyu Dong ${ }^{4}$, Dezhi Li ${ }^{5}$, Kun Shi ${ }^{4}$ and \\ Haibo Zhang 6 \\ 1 College of Information and Electrical Engineering, China Agricultural University, Haidian District, \\ Beijing 100083, China; wangjiankai@cau.edu.cn \\ 2 Linyi Power Supply Company, State Grid Shandong Electric Power Company, Linyi 276000, China; \\ gefuchao@cau.edu.cn \\ 3 Power Planning Department State Grid Fujian Economic Research Institute, Fuzhou 350000, China; \\ jyy.lin_yi@fj.sgcc.com.cn \\ 4 Beijing Key Laboratory of Demand Side Multi-Energy Carriers Optimization and Interaction Technique, \\ Beijing 100192, China; dongmingyu@epri.sgcc.com.cn (M.D.); shikun@epri.sgcc.com.cn (K.S.) \\ 5 China Electric Power Research Institute, Haidian District, Beijing 100192, China; lidezhi@epri.sgcc.com.cn \\ 6 The State Key Laboratory of Alternate Electrical Power System with Renewable Energy Sources, \\ North China Electric Power University, Beijing 102206, China; zhb@ncepu.edu.cn \\ * Correspondence: juyuntao@cau.edu.cn
}

Received: 24 March 2019; Accepted: 12 April 2019; Published: 18 April 2019

check for updates

\begin{abstract}
As more clean energy sources contribute to the electrical grid, the stress on generation scheduling for peak-shaving increases. This is a concern in several provinces of China that have many nuclear power plants, such as Guangdong and Fujian. Studies on the unit commitment (UC) problem involving the characteristics of both wind and nuclear generation are urgently needed. This paper first describes a model of nuclear power and wind power for the UC problem, and then establishes an objective function for the total cost of nuclear and thermal power units, including the cost of fuel, start-stop and peak-shaving. The operating constraints of multiple generation unit types, the security constraints of the transmission line, and the influence of non-gauss wind power uncertainty on the spinning reserve capacity of the system are considered. Meanwhile, a model of an energy storage system (ESS) is introduced to smooth the wind power uncertainty. Due to the prediction error of wind power, the spinning reserve capacity of the system will be affected by the uncertainty. Over-provisioning of spinning reserve capacity is avoided by introducing chance constraints. This is followed by the design of a UC model applied to different power sources, such as nuclear power, thermal power, uncertain wind power, and ESS. Finally, the feasibility of the UC model in the scheduling of a multi-type generation unit is verified by the modified IEEE RTS 24-bus system accommodating large scale green generation units.
\end{abstract}

Keywords: nuclear power generation; wind power generation; energy storage system; unit commitment; Gaussian mixture model; spinning reserve

\section{Introduction}

Rapid growth in electrification together with more diversified clean power generation is causing an increasing disparity between peak and valley energy demands. In addition, there is a growing trend to integrate clean energy sources, like solar, wind and nuclear power, into the power grid. Most of China's nuclear power plants are distributed in coastal areas, and their capacity is still increasing. The uncertainty of wind power and inflexible nuclear power generation means that the power grid frequency fluctuates easily and aggravates the burden of power system peak-shaving. Therefore, it is 
urgent to study how to schedule a multi-type generation unit that includes nuclear power and wind power for peak system operation.

Nuclear power plants are one of the key clean energy sources in China. In particular, the proportion of nuclear power in coastal provinces such as Fujian and Guangdong is still increasing. For safe operation, nuclear generation units usually operate at an invariant power to support the basic load and do not participate in load tracking or peak-shaving.

At present, nuclear power plants in the United States, Japan, and France are participating in peak-shaving. In [1], the feasibility and necessity of nuclear power plants participating in peak-shaving of power systems are described, including analysis of the modes and characteristics of peak operation. Fang et al. analyzed the feasibility of controlling the power of a pressurized water reactor nuclear power unit through the control rod in [2]. The core simulation and operating characteristics of AP1000 were studied in [3]. In [4], the ability and characteristics of peak-shaving with various nuclear generation units were analyzed, and existing problems in nuclear power generation and strategies for joint peaking operation of the other power sources were described. In [5], the advantage of nuclear power generation participation in load-following was studied, and it was shown that a nuclear power unit can directly participate in the system's daily load peak-shaving based on the "12-3-6-3" mode, as verified by actual data from the grid.

In summary, there are few studies on joint optimization scheduling with nuclear power, non-Gaussian wind power generation, and energy storage. In [6], thermal and water joint optimization generation was proposed with the aim of minimizing the total power generation system cost, while also considering the constraints of pollutant emission and implementing suitable safety measures. In [7], the authors considered the peak regulation features of a security operation for nuclear power generation and used an objective function to quantify the total cost of thermal-nuclear-pumped storage; however, the integration of non-gaussian uncertain wind power units was not considered.

The use of ESS can mitigate some of the problems associated with the unpredictable nature of wind power generation [8]. ESS can function as a virtual power generation device by implementing peak-shaving and load following by absorbing and discharging energy to meet the demands of the grid. Energy storage technology, such as super conducting magnetic energy storage [9], thermal electric energy storage [10] and batteries [11] is currently developing rapidly. Large-scale ESSs have been considered for joint optimization scheduling [12,13].

The uncertainty associated with wind power causes difficulties in effective scheduling. A certain number of spinning reserves is needed in power generation plans to handle the problems of wind power and load uncertainty. Generally, reserve capacity is determined by a load demand ratio. However, given the non-gaussian distribution of prediction error of wind power, the reliability and economy needs of the power system cannot be guaranteed. There are two approaches to handle the unit commitment (UC) models associated with wind power prediction error. First, according to the uncertainty and probability distribution of wind power output, the system's spin reserve capability and confidence interval can be set, respectively [14,15]. Multi-scene technology can be applied by analyzing the influence of wind power prediction error on generation scheduling results by simulating the discrete scenarios of various wind power output uncertainties [16-18]. However, this is a computationally expensive procedure.

The conventional UC problem associated with thermal power is a lack of features that can be integrated with nuclear power and non-gaussian distributed random wind power combination participate in joint peak-shaving. The spinning reserve capacity constraints considering uncertainty and transmission line thermal security are derived with chance constraint conditions. The wind power forecast error will affect the system's reserve capacity uncertainty. Introducing a spinning reserve chance constraint considering non-Gaussian wind power generation decreases redundant over-provisioning. The ESS for a power station has been designed in the form of a scheduling optimization model. Ultimately, a joint optimization model involving multi-type generation units is 
built and optimal day-ahead generation unit scheduling strategies are given and compared under different situations.

\section{Modeling of Nuclear and Wind Power}

\subsection{UC Modeling of Nuclear Power Generation}

Load following control of a nuclear power plant is obtained by regulating the nuclear reactor power. Generally, through controlling the concentration of the boron solution and the rod displacement height, load following control of the reactor is achieved.

At present, most nuclear power plants in China are second generation pressurized water reactors (PWRs). They have the capacity to participate in daily load tracking. Considering the restrictions imposed by nuclear power ramp rate constraints and peak-shaving depth constraints, the PWR nuclear power units can participate in daily peak-shaving in the "12-3-6-3" power output mode, i.e., they operate at rated power for $12 \mathrm{~h}$, then drop to a light-load power level after $3 \mathrm{~h}$, continue to run at lower power sustaining for $6 \mathrm{~h}$, and then rise to rated power in $3 \mathrm{~h}$ before entering the next cycle. The peak-shaving mode of "12-3-6-3" is shown in Figure 1.

In addition, with the development of nuclear power technology, AP1000, EPR and other three generations of nuclear power units show more effective regulation performance, and participate in daily load tracking; peak-shaving operation is also more flexible. They can achieve "15-1-7-1" peak-shaving operation mode within $90 \%$ of the operating lifetime of the unit, as shown in Figure 1.

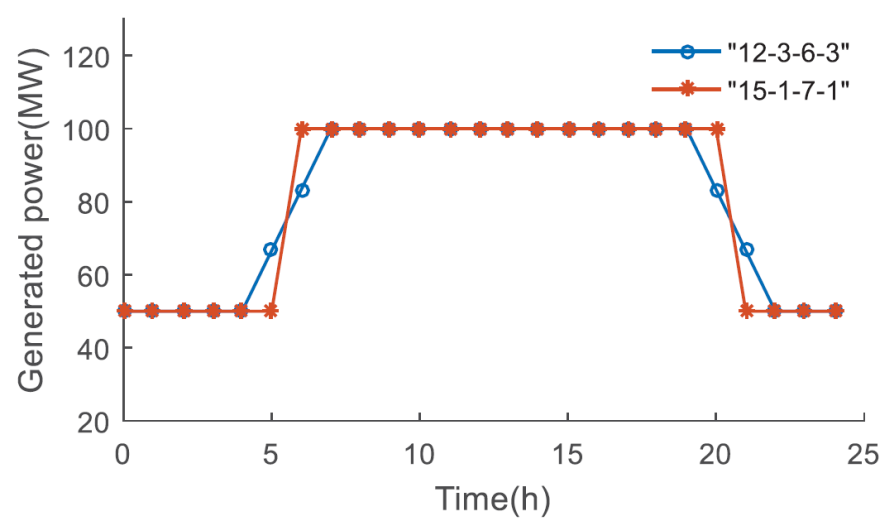

Figure 1. Two nuclear power generation load following modes.

The nuclear power output model with peak-shaving characteristics should be considered in the UC problem. The mathematical model of the output power is expressed as [7]:

$$
P_{N, i t}=g_{i t}\left(P_{N, i \min }+\Delta P_{N, i}\right)+h_{i t}\left(P_{N, i \min }+2 \Delta P_{N, i}\right)+e_{i t} P_{N, i \max }+f_{i t} P_{N, i \text { min }}
$$

where,

$$
\begin{aligned}
& e_{i t}+f_{i t}+g_{i t}+h_{i t}=1 \\
& e_{i k} \geq e_{i t}-e_{i t-1} \quad k=t, t+1, \ldots, t+T_{i}^{e}-1 \\
& f_{i k} \geq f_{i t}-f_{i t-1} \quad k=t, t+1, \ldots, t+T_{i}^{f}-1 \\
& h_{i t+1} \geq f_{i t}+g_{i t-1}-1 \\
& e_{i t+1} \geq h_{i t}+g_{i t-1}-1 \\
& g_{i t+1} \geq h_{i t}+e_{i t-1}-1 \\
& f_{i t+1} \geq g_{i t}+h_{i t-1}-1
\end{aligned}
$$

where, $e_{i t}, f_{i t}, g_{i t}, h_{i t}$ are all $\{0,1\}$ variables, and denote the operation state index with respect to four nuclear power operating levels: $T_{i}^{e}$ and $T_{i}^{f}$ are the minimum running time of the rated power and the 
low power modes, respectively; $P_{N, i \text { max }}, P_{N, i \text { min }}$ are the maximum and minimum injected power; and $\Delta P_{N, i}$ represents the power variation of a nuclear generation unit $i$ within $1 \mathrm{~h}$.

Under rapid variation of generation, traditional thermal power generation, creep fatigue may come up in the main steam line. Meanwhile, service life of water pump, deaerator, and high pressure heater will be reduced. In general, deep peak-shaving of nuclear power plant is arranged at the $65 \%$ of fuel capacity. After, the depth of peak-shaving will be lowered. Under rapid variation of nuclear generation, clad shell may bear limiting stress. Service life of nuclear generation will be reduced.

\subsection{Modeling of Wind Power Generation Uncertainty}

The random and fluctuating characteristics of wind power make it difficult to predict, and therefore plan, flexible scheduling. The analysis of wind power uncertainty is of great significance to UC models of wind farms. The forecast error of wind power output is expressed as:

$$
P_{w}=P_{f}+\Delta P_{e}
$$

where, $P_{w}$ represents the actual power, $P_{f}$ denotes the forecasted wind power, and $\Delta P_{e}$ represents the forecast error.

In theory, the forecast errors of wind power belong to Gaussian distribution. Due to the different forecast methods, time scales and geographical environments of wind farms, the probability distribution of the forecast error shows different distribution, non-universal characteristics.

The Gaussian mixture model (GMM) is a linear combination of single Gaussian probability density functions. It can accurately describe the probability density distribution of various shapes by adjusting either the linear combination weights or the parameter estimates. The Gaussian mixture distribution makes the forecast error modeling of wind power more accurate. The forecast error of wind power is regarded as a random variable subject to Gaussian mixture distribution, and then the probabilistic constraint model is transformed into the deterministic constraint model and solved using the chance constraint programming theory. A detailed solution is provided in the Appendix A.

The variance can be solved by the clustering algorithm [19]; the equivalent mean and covariance are expressed as:

$$
\begin{gathered}
\omega_{m}=\sum_{j \in \mathrm{I}} \omega_{j} \\
\mu_{m}=\frac{1}{\omega_{m}} \sum_{j \in \mathrm{I}} \omega_{j} \mu_{j} \\
\sigma_{m}=\frac{1}{\omega_{m}} \sum_{j \in \mathrm{I}} \omega_{j}\left[\sigma_{j}+\left(\mu_{j}-\mu_{m}\right)\left(\mu_{j}-\mu_{m}\right)^{T}\right]
\end{gathered}
$$

where, $\omega_{j}$ and $\omega_{m}$ are the weight of the $j$ th mixture component and the total weight, respectively, $\mu_{j}$ and $\mu_{m}$ are the mean of the $j$ th mixture component and the total mean, respectively, $\sigma_{j}$ and $\sigma_{m}$ are the standard deviation of the $j$ th mixture component and the total standard deviation, respectively, and $T$ is determined by the $\chi^{2}$-test with $99 \%$ confidence.

Prediction accuracy is improving with the development of new technology. It is assumed that the forecast errors of load and wind power are subject to Gaussian distribution and Gaussian mixture distribution, respectively. Here, $\sigma_{L}, \sigma_{W}$ denote the standard deviation of the forecast error distribution of load and wind power, respectively $[16,20]$. $\sigma_{W}$ can be solved by Formulas (3)-(5). Assuming that the load and wind power forecast errors are uncorrelated random variables, the standard deviation of the total forecast error can be expressed as [21]:

$$
\sigma=\sqrt{\left(\sigma_{L}^{t}\right)^{2}+\left(\sigma_{W}^{t}\right)^{2}}
$$


Considering the uncertain factors, additional spinning reserve capacity is needed to ensure the security of generation scheduling. The spinning reserve capacity is mainly from thermal generation units, and the spinning reserve capacity for total power system at each time scale is expressed as:

$$
\sum_{i=1}^{m_{0}} u_{i, t} P_{G, i \max }-\sum_{i=1}^{m_{0}} P_{G, i t} \geq \operatorname{Res}_{t}
$$

where Res $s_{t}$ denotes the spinning reserve capacity at time $t$.

$\tilde{P}_{i, t}^{W}$ and $\tilde{P}_{L, t}$ are introduced, which are equal to the expected values $P_{i, t}^{W}$ and $P_{L, t}$ plus the error values $\Delta P_{i, t}^{W}$ and $\Delta P_{L, t}$, respectively. Formula (7) is expressed as:

$$
\sum_{i=1}^{m_{0}} u_{i, t} P_{G, i \max }-\sum_{i=1}^{m_{0}} P_{G, i t} \geq \operatorname{Res}_{t}+\sum_{i=1}^{m_{2}} \Delta P_{i, t}^{W}+\Delta P_{L, t}
$$

Here, $z_{t} \triangleq \sum_{i=1}^{m_{2}} \Delta P_{i, t}^{W}+\Delta P_{L, t}$ is a random variable, and $F_{z_{t}}^{-1}(\cdot)$ denotes the inverse of the cumulative distribution function. With application of the chance constraints, (8) is written by:

$$
P\left(z_{t} \leq \sum_{i=1}^{m_{0}}\left(u_{i, t} P_{G, i \max }-P_{G, i t}\right)-\operatorname{Res}_{t}\right) \geq \alpha
$$

Then, the conversion of probability constraints and deterministic constraints is achieved by $F_{z_{t}}^{-1}(\cdot)$.

$$
\sum_{i=1}^{m_{0}}\left(u_{i, t} P_{G, i \max }-P_{G, i t}\right)-\operatorname{Res}_{t} \geq F_{z_{t}}^{-1}(\alpha)
$$

where $\alpha$ denotes the confidence level.

According to the standard deviation of the total forecast error (6), (10) is further approximated as:

$$
\sum_{i=1}^{m_{0}}\left(u_{i, t} P_{G, i \max }-P_{G, i t}\right) \geq \operatorname{Res}_{t}+\eta(\alpha)\left(\left(\sigma_{D}^{t}\right)^{2}+\left(\sigma_{W}^{t}\right)^{2}\right)^{1 / 2}
$$

where $\eta(\alpha)$ denotes cumulative distribution function at confidence level $\alpha$, which can be acquired by looking up the Gaussian distribution table.

\section{UC Program Considering Multiple Generation Types}

The UC program involving various generation types considers the constraints of each generation unit's operation characteristics together with the security constraints of the whole system. The optimization goal is to minimize the operating cost of the grid by determining the operational status of each generation unit. This paper mainly considers thermal power, wind power, nuclear power, and energy storage.

\subsection{Objective of UC}

The objective of UC is to minimize the operating cost of the whole system, which mainly comprises thermal and nuclear power expenses.

$$
\begin{aligned}
\min O F= & \sum_{t=1}^{T} \sum_{i=1}^{m_{0}}\left(F C_{G, i t}+S T C_{G, i t}+S D C_{G, i t}\right) \\
& +\sum_{t=1}^{T} \sum_{i=1}^{m_{0}} g_{G, i} \cdot C_{G}+\sum_{t=1}^{T} \sum_{i=1}^{m_{1}} F C_{N, i t} \\
& +\sum_{t=1}^{T} \sum_{i=1}^{m_{1}}\left(P_{N, i \max }-P_{N, i t}\right) \cdot C_{N}
\end{aligned}
$$

where, $T$ represents the time horizon in one day (i.e., $T=24 \mathrm{~h}$ ), $t$ denotes the time period (hours), and $m_{0}$ and $m_{1}$ denote the number of thermal power units and nuclear power units, respectively. $F C_{N, i t}$ is the fuel cost of the nuclear power units, $F C_{G, i t}=a_{i} P_{G, i t}^{2}+b_{i} P_{G, i t}+c_{i}$ is the quadratic function, which represents the fuel cost of thermal power units; $S T C_{G, i t}=s t_{i} y_{i t}$ and $S D C_{G, i t}=$ 
$s d_{i} z_{i t}$ represent the up/down cost of thermal generation units, respectively, $\sum_{t=1}^{T} \sum_{i=1}^{m_{0}} g_{G, i} \cdot C_{G}$ and $\sum_{t=1}^{T} \sum_{i=1}^{m_{1}}\left(P_{N, i \max }-P_{N, i t}\right) \cdot C_{N}$ denote the peak-shaving costs of thermal power units and nuclear power units, respectively; and $a_{i}, b_{i}, c_{i}, C_{G}, C_{N}, s t_{i}, s d_{i}$ denote the known cost coefficients of generators.

There, the quadratic function of the fuel cost should be linearized. Firstly, the interval $\left[P_{i}^{\min }, P_{i}^{\max }\right]$ is divided into $n$ equal-sized subintervals. Secondly, for a particular subinterval $k$, we define a variable $p_{i, t}^{k}$ that varies between zero and the subinterval length $\Delta P_{i}^{k}$. Furthermore, the relevant parameters of subinterval $k$ should satisfy the following constraints.

$$
\begin{aligned}
& 0 \leq p_{i, t}^{k} \leq \Delta P_{i}^{k} u_{i, t} \quad \forall k=1: n \\
& \Delta p_{i}^{k}=\frac{P_{i}^{\max }-P_{i}^{\min }}{n} \\
& P_{i, \text { ini }}^{k}=(k-1) \Delta P_{i}^{k}+P_{i}^{\min } \\
& P_{i, \text { fin }}^{k}=\Delta P_{i}^{k}+P_{i, \text { ini }}^{k} \\
& P_{i, t}=P_{i}^{\min } u_{i, t}+\sum_{k} p_{i, t}^{k} \\
& C_{i, \text { ini }}^{k}=a_{i}\left(P_{i, \text { int }}^{k}\right)^{2}+b_{i} P_{i, \text { int }}^{k}+c_{i} \\
& C_{i, \text { fin }}^{k}=a_{i}\left(P_{i, \text { fin }}^{k}\right)^{2}+b_{i} P_{i, \text { fin }}^{k}+c_{i} \\
& s_{i}^{k}=\frac{C_{i, \text { fin }}^{k}-C_{i, \text { ni }}^{k}}{\Delta P_{i}^{k}}
\end{aligned}
$$

where, $s_{i}^{k}$ is the slope of the line segment between the start point and the end point in the subinterval $k$.

Finally, linear expression of the quadratic function of the fuel cost is as follows.

$$
F C_{i, t}=\left(a_{i}\left(P_{i}^{\min }\right)^{2}+b_{i} P_{i}^{\min }+c_{i}\right) u_{i, t}+\sum_{k} s_{i}^{k} p_{i, t}^{k}
$$

\subsection{Constraints for Generation Units}

The constraints of the conventional UC program include network safety, power balance and minimum up/down time, etc. If the nuclear power, wind power and the energy storage participate in day-ahead-scheduling, the impact of nuclear power plant peak-shaving operation characteristics and wind power uncertainty on spinning reserve should be considered. These constraints are expressed as follows.

\subsubsection{Load and Generation Power Balance Constraints}

The power balance constraints are expressed as:

$$
\sum_{i=1}^{m_{0}} P_{G, i t}+\sum_{i=1}^{m_{1}} P_{N, i t}+\sum_{i=1}^{m_{2}} P_{i, t}^{W}=P_{L, t}
$$

where, $P_{G, i t}$ and $P_{N, i t}$ represent the output power of the thermal generation unit and the nuclear power at time $t$, respectively, $P_{i, t}^{W}$ represents the predicted power of the wind generation unit $i$ at time $t$, and $P_{L, t}$ represents the system's total load demand at time $t$.

\subsubsection{Minimum and Maximum Injected Power Constraints}

The minimum and maximum injected power constraints are expressed as:

$$
u_{i, t} P_{G, i \min } \leq P_{G, i t} \leq u_{i, t} P_{G, i \max }
$$

where, $P_{G, i \min }$ and $P_{G, i \max }$ represent the minimum power generation and maximum power generation of the thermal power unit $i$, respectively; $u_{i, t}$ represents the operating status of unit $i$ at time $t$; and $u_{i, t}=0 / 1$ represents that unit $i$ is in the off/on state at time $t$. 


\subsubsection{Reserve Capacity Constraints}

According to Section 2.2, the spinning reserve capacity considering both the load and wind power prediction uncertainty is expressed as:

$$
\sum_{i=1}^{m_{0}}\left(P_{G, i \max }-P_{G, i t}\right)+\sum_{i=1}^{m_{1}}\left(P_{N, i \text { max }}-P_{N, i t}\right) \geq \operatorname{Res}_{t}+\eta(\alpha)\left(\left(\sigma_{D}^{t}\right)^{2}+\left(\sigma_{W}^{t}\right)^{2}\right)^{1 / 2}
$$

\subsubsection{Ramp Rate Constraints}

In order to cope with changes in load and wind power output, thermal power units need to adjust their output in time. The ramp rate constraints are expressed as:

$$
\begin{aligned}
& P_{i t}-P_{i t-1} \leq R U_{i} \\
& P_{i t-1}-P_{i t} \leq R D_{i}
\end{aligned}
$$

where, $R D_{i}$ and $R U_{i}$ denote the down/up active power limit of unit $i$ under normal operating status, respectively.

\subsubsection{Minimum Thermal Generation up/down Time Constraints}

By discretizing the nonlinear startup cost, we can get a piecewise linear function. The formula of the minimum up time is expressed as [22]:

$$
\begin{gathered}
\sum_{t=1}^{\zeta_{i}} 1-u_{i, t}=0 \\
\sum_{t=k}^{k+U T_{i}-1} u_{i, t} \geq U T_{i} y_{i, t}, \quad \forall k=\zeta_{i}+1 \ldots T-U T_{i}+1 \\
\sum_{t=k}^{T} u_{i, t}-y_{i, t} \geq 0, \quad \forall k=T-U T_{i}+2 \ldots T \\
\zeta_{i}=\min \left\{T,\left(U T_{i}-U_{i}^{0}\right) u_{i, t=0}\right\}
\end{gathered}
$$

The formula of the minimum down time is expressed as:

$$
\begin{gathered}
\sum_{t=1}^{\xi_{i}} u_{i, t}=0 \\
\sum_{t=k}^{k+D T_{i}-1} 1-u_{i t} \geq U T_{i} z_{i, k}, \forall k=\xi_{i}+1 \ldots T-D T_{i}+1 \\
\sum_{t=k}^{T} 1-u_{i, t}-z_{i, t} \geq 0, \forall k=T-D T_{i}+2 \ldots T \\
\xi_{i}=\min \left\{T,\left(D T_{i}-S_{i}^{0}\right)\left(1-u_{i, t=0}\right)\right\}
\end{gathered}
$$

where, $D T_{i}$ and $U T_{i}$ denote the minimum down/up time of thermal generation unit $i$, respectively; $U_{i}^{0}$ and $S_{i}^{0}$ denote the initial down/up time, respectively; and $y_{i, t}$ and $z_{i, t}$ denote the start-up/shut-down status, respectively. The constraint that $y_{i, t}$ and $z_{i, t}$ satisfy is expressed as:

$$
\begin{gathered}
y_{i, t}-z_{i, t}=u_{i, t}-u_{i, t-1} \\
y_{i, t}+z_{i, t} \leq 1 \\
y_{i, t}, z_{i, t}, u_{i, t} \in\{0,1\}
\end{gathered}
$$

\subsubsection{Nuclear Power Peak Regulation Depth Constraints}

The nuclear power peak-shaving depth constraints are expressed as:

$$
\eta_{i}=\frac{P_{N, i \max }-P_{N, i}}{P_{N, i \max }} \leq \eta_{\max }
$$

where, $\eta_{i}$ represents peak-shaving depth, $P_{N, i}$ denotes power generation of nuclear plant $i, P_{N, i \max }$ is maximum power generation of nuclear plant, i.e., the capacity of nuclear plant. $\eta_{\max }$ is maximum peak-shaving depth. For example, the capacity of nuclear plant is $100 \mathrm{MW}$, the maximum peak-shaving of $0.8, P_{N, i}$ can not be lower than $20 \mathrm{MW}$. 
The nuclear generation unit has various installed capacities, reactor types and thresholds, $\eta_{\max }$, of maximum peak-shaving depth.

The constraint to be satisfied during depth peak-shaving of the thermal power unit is expressed as:

$$
\begin{gathered}
P_{G, i \text { min }}^{\prime} \leq P_{G, i t} \leq P_{G, i \text { max }} \\
g_{G, i t}= \begin{cases}P_{G, i \text { min }}^{\prime}-P_{G, i t} & P_{G, i t}<P_{G, i \text { min }}^{\prime} \\
0 & P_{G, i t} \geq P_{G, i \text { min }}^{\prime}\end{cases}
\end{gathered}
$$

The basic and depth peak-shaving states are two states in which thermal power participates in peak-shaving. The injected power threshold is denoted by $P_{G, i \text { min }}^{\prime}$, which is used to identify the two states, i.e., peak-shaving cost compensation is only given when a certain peak-shaving depth is reached. Where, $P_{G, i \text { min }}^{\prime}$ is the minimum limit of the injected power of the thermal generation that taking part in the basic peak-shaving; and $P_{G, i t}$ is the injected active power with respect to the thermal generation unit $i$ at time $t$.

\subsubsection{Load Following Constraints of Nuclear Generation Units}

According to Section 2.1, the nuclear generation unit considering the peak-shaving characteristics is expressed as:

$$
P_{N, i t}=e_{i t} P_{N, i \text { max }}+f_{i t} P_{N, i \text { min }}+g_{i t}\left(P_{N, i \text { min }}+\Delta P_{N, i}\right)+h_{i t}\left(P_{N, i \text { min }}+2 \Delta P_{N, i}\right)
$$

\subsubsection{ESS Constraints}

The ESS cannot exceed the maximum and minimum limits capacity:

$$
\begin{gathered}
S O C_{i, t}=S O C_{i, t-1}+\left(P_{i, t}^{c} \eta_{c}-P_{i, t}^{d} / \eta_{d}\right) \Delta t \\
S O C_{\text {min }} \leq S O C_{t} \leq S O C_{\text {max }}
\end{gathered}
$$

where, $S O C_{\max }$ and $S O C_{\min }$ denote the maximum and minimum limits of the residual capacity of the ESS, respectively; $S O C_{t}$ is the storage capacity at time $t . P_{i, t}^{c}$ and $P_{i, t}^{d}$ denote the charge/discharge power at time $t$, respectively, $\eta_{c}$ denotes the charging efficiency; and $\eta_{d}$ represents discharging efficiency; $\Delta t$ denotes the interval time scale, is equal to $1 \mathrm{~h}$.

Considering the current limitations of converters, the injected power of the ESS at each moment cannot violate a certain power limit.

$$
\begin{aligned}
& P_{i, \text { min }}^{c} \leq P_{i, t}^{c} \leq P_{i, \text { max }}^{c} \\
& P_{i, \text { min }}^{d} \leq P_{i, t}^{d} \leq P_{i, \text { max }}^{d}
\end{aligned}
$$

where $P_{i, \max }^{c}$ and $P_{i, \max }^{d}$ denote the maximum active power limits during charging and discharging respectively. Here, the lower limits we set are $P_{i, \text { min }}^{c}=P_{i, \min }^{d}=0, P_{i, \max }^{c}=P_{i, \max }^{d}=S O C_{\max }$.

Besides, the depth and timing of charge and discharge affect the life of the battery; relevant constraint models need to be introduced for studying their influence on battery life.

\subsubsection{Line Transmission Power Constraints}

The line transmission power constraints are expressed as:

$$
\begin{gathered}
\sum_{\mathrm{g} \in \Omega_{G}^{i}} P_{g, t}+\sum_{h \in \Omega_{H}^{i}} P_{h, t}+P_{i, t}^{w}-L_{i, t}-P_{i, t}^{c}+P_{i, t}^{d}=\sum_{j \in \Omega_{\ell}^{i}} P_{i j, t} \\
P_{i j, t}=\frac{\theta_{i, t}-\theta_{j, t}}{x_{i j}}
\end{gathered}
$$




$$
-P_{i j, t}^{\max } \leq P_{i j, t} \leq P_{i j, t}^{\max }
$$

where $\theta_{i, t}$ denotes bus $i$ voltage angle at time $t$, and $x_{i j}$ denotes the branch reactance between bus $i$ and bus $j$. $P_{i j, t}^{\max }$ represents the maximum active power flow limits for branch $i j . \Omega_{G}^{i}$ represents all thermal generation units at bus $i, \Omega_{H}^{i}$ represents all nuclear power units at bus $i$, and $\Omega_{\ell}^{i}$ represents all buses linked to bus $i$.

Introducing the probability constraint, the line transmission capacity constraint is the joint opportunity constraint when the line power is regarded as a random variable.

$$
F C_{i, t}=\left(a_{i}\left(P_{i}^{\min }\right)^{2}+b_{i} P_{i}^{\min }+c_{i}\right) u_{i, t}+\sum_{k} s_{i}^{k} p_{i, t}^{k}
$$

Equation (32) is transformed into a deterministic constraint as follows:

$$
\begin{aligned}
& P_{i j, t} \leq P_{i j, t}^{\max }-F_{\Delta P_{i j, t}}^{-1}\left(\beta_{1}\right) \\
& P_{i j, t} \geq P_{i j, t}^{\min }+F_{\Delta P_{i j, t}^{-1}}\left(\beta_{2}\right)
\end{aligned}
$$

where $\beta_{1}$ and $\beta_{2}$ are the confidence level.

\section{Case Study}

The effectiveness of the proposed UC model is verified by the modified IEEE RTS-24 bus system, which consists of 10 thermal generation units, two wind farms, two nuclear power stations and two energy storage power stations. The cost coefficients of the generators are listed in Tables 1 and 2. The daily load demand and wind power generation are shown in Figure 2. The quadratic function of the fuel cost and the constraints of minimum up/down time are linearized by reference to the literature [22]. The proposed UC mathematical model is solved with GAMS MINLP solver.

Table 1. Cost coefficients of thermal power units.

\begin{tabular}{cccccc}
\hline Number & $\boldsymbol{a}_{\boldsymbol{i}}\left(\mathbf{\$} / \mathbf{M W}^{\mathbf{2}} \mathbf{h}\right)$ & $\boldsymbol{b}_{\boldsymbol{i}}(\mathbf{\$} / \mathbf{M W h})$ & $\boldsymbol{c}_{\boldsymbol{i}}(\mathbf{\$} / \mathbf{h})$ & $\boldsymbol{s t _ { i } ( \mathbf { } )}$ & $\boldsymbol{s} \boldsymbol{d}_{\boldsymbol{i}}(\mathbf{\$})$ \\
\hline g1 & 0.00048 & 16.19 & 1000 & 52,000 & 13,000 \\
g2 & 0.00048 & 16.19 & 1000 & 52,000 & 13,000 \\
g3 & 0.0021 & 16.8 & 720 & 17,667 & 4367 \\
g4 & 0.0021 & 16.8 & 720 & 17,667 & 4367 \\
g5 & 0.002 & 16.6 & 700 & 17,667 & 4367 \\
g6 & 0.002 & 16.6 & 700 & 16,667 & 4167 \\
g7 & 0.001 & 16.19 & 800 & 50,000 & 12,000 \\
g8 & 0.00068 & 16.19 & 850 & 50,000 & 12,000 \\
g9 & 0.00068 & 16.19 & 850 & 50,000 & 12,000 \\
g10 & 0.001 & 16.19 & 800 & 0 & 0 \\
\hline
\end{tabular}

Table 2. Cost coefficients of nuclear power units.

\begin{tabular}{ccccc}
\hline Number & $\boldsymbol{a}_{\boldsymbol{i}}\left(\mathbf{\$} / \mathbf{M W}^{\mathbf{2}} \mathbf{h}\right)$ & $\boldsymbol{b}_{\boldsymbol{i}}(\mathbf{\$} / \mathbf{M W h})$ & $\boldsymbol{c}_{\boldsymbol{i}}(\mathbf{\$} \mathbf{h})$ & $\boldsymbol{P}_{\boldsymbol{N}, \mathbf{i} \text { Max }}(\mathbf{})$ \\
\hline $\mathrm{h} 1$ & 0 & 9.33 & 7320 & 300 \\
$\mathrm{~h} 2$ & 0 & 9.33 & 7320 & 100 \\
\hline
\end{tabular}

The total installed capacity of the wind farms connected to lines 8 and 21 is 200 and $50 \mathrm{MW}$, respectively. The installed capacity of the nuclear power plant connected to buses 1 and 18 is 100 and $300 \mathrm{MW}$, respectively.

In the test system, the wind generation is linked to both bus 8 and bus 21. The Gaussian mixture parameters of wind power forecast error are $c_{1}^{(1)}=0.7, u_{1}^{(1)}=0, \sigma_{1}^{(1)}=8, c_{2}^{(1)}=0.3, u_{2}^{(1)}=4, \sigma_{2}^{(1)}=2$, $c_{1}^{(2)}=0.8, u_{1}^{(2)}=1, \sigma_{1}^{(2)}=4, c_{2}^{(2)}=0.2, u_{2}^{(2)}=5, \sigma_{2}^{(2)}=10$. On lines 12 and 13 , the corresponding wind turbine output power transfer distribution factors $\beta^{(1)}$ and $\beta^{(2)}$ are 0.26 and 0.42 , respectively. The detailed derivation of line transfer power probability is provided in the Appendix A. 

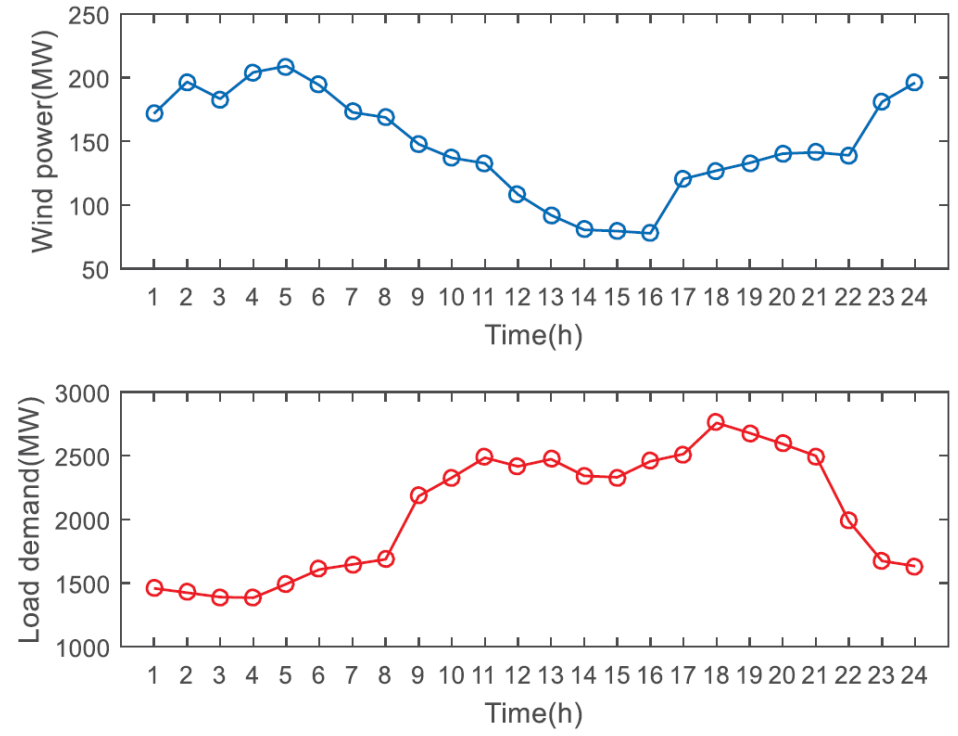

Figure 2. The hourly load demand and wind power prediction.

\subsection{Impact on Cost of Peak Regulation with Nuclear Power}

In this part, three schemes are designed for comparative analysis.

(1) Scheme one

Nuclear power units do not participate in peak-shaving scheduling and maintain stable operation at full power.

\section{(2) Scheme two}

Nuclear power units participate in peak-shaving scheduling of the power system according to the output pattern of "12-3-6-3". The peak regulation depth of two nuclear power units is set in advance at $30 \%$.

(3) Scheme three

The method of peak-shaving scheduling of the power system is the same as in scheme two. The optimization solution is based on the joint optimal peak-shaving scheduling model. The optimization cost results are shown in Figure 3.

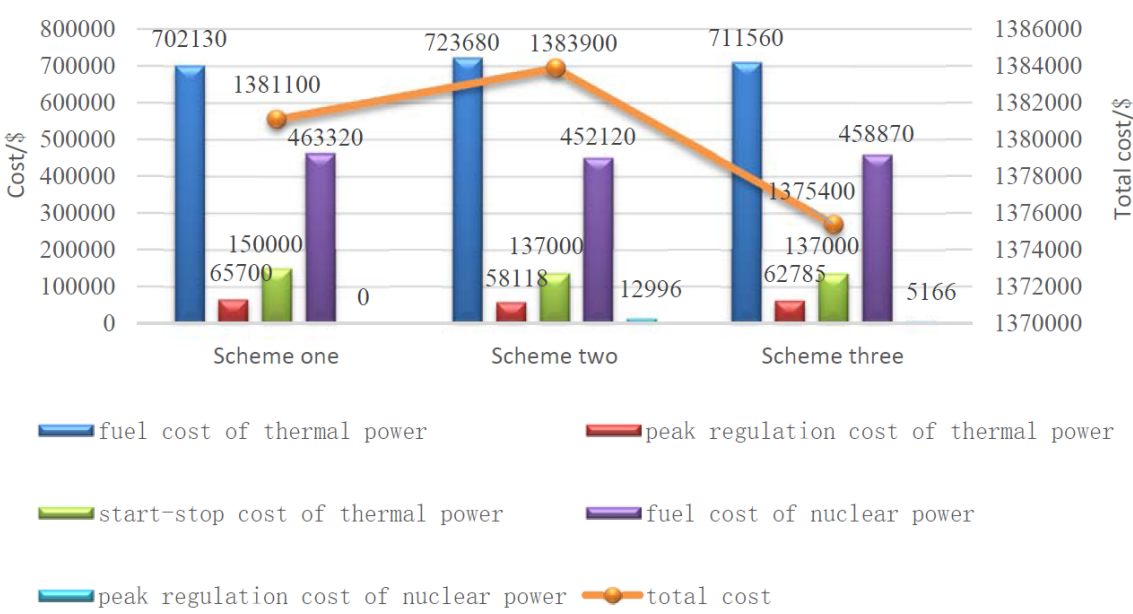

Figure 3. Cost comparison among peak regulation operation types. 
As can be seen in Figure 3, scheme three has the lowest total operating cost. At this time, the optimal peak-shaving depth of the two nuclear power units is $0 \%$ and $29.8 \%$, respectively. Shorter start-stop operation times are the main reason for the reduced operational costs. For example, compared to scheme one, the one-time stop operation cost of thermal power unit 3 (g3) is reduced in schemes two and three with peak-shaving of nuclear power units.

\subsection{Optimal Scheduling of the Proposed Multi-Type Generation Unit}

Considering the joint dispatching model presented, including nuclear generation, the optimized peak shaving depth of nuclear power is set to $\eta_{i}=30 \%$, The injected power and up/down status of 10 thermal power units are shown in Figure 4, where we can see that the No. 5 and No. 6 thermal power units (g5 and g6, respectively) are always in a stop state, which avoids the high up/down cost.

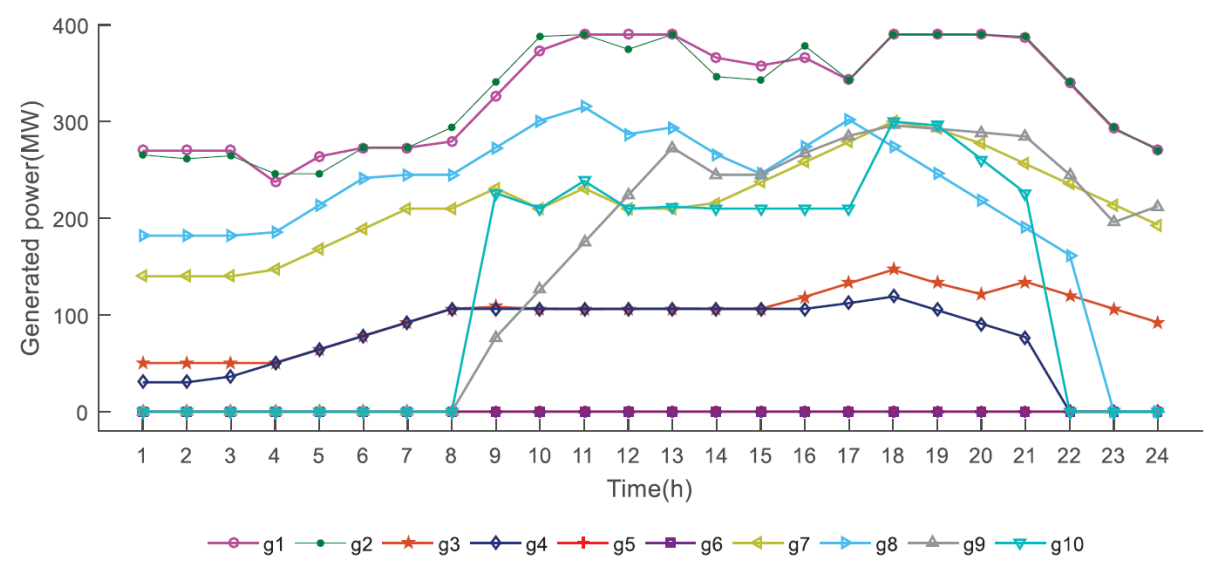

Figure 4. Injected active power of thermal generation units.

The optimized peak regulation output curves of the No. 1 and No. 2 nuclear power plants (h1 and h2) are shown in Figure 5, respectively. The output of nuclear generation follows the system demand, which effectively decreases the peak-valley load distinction. Figure 6 shows the charging and discharging active power of ESS on both bus 8,21, and the total power at each time.
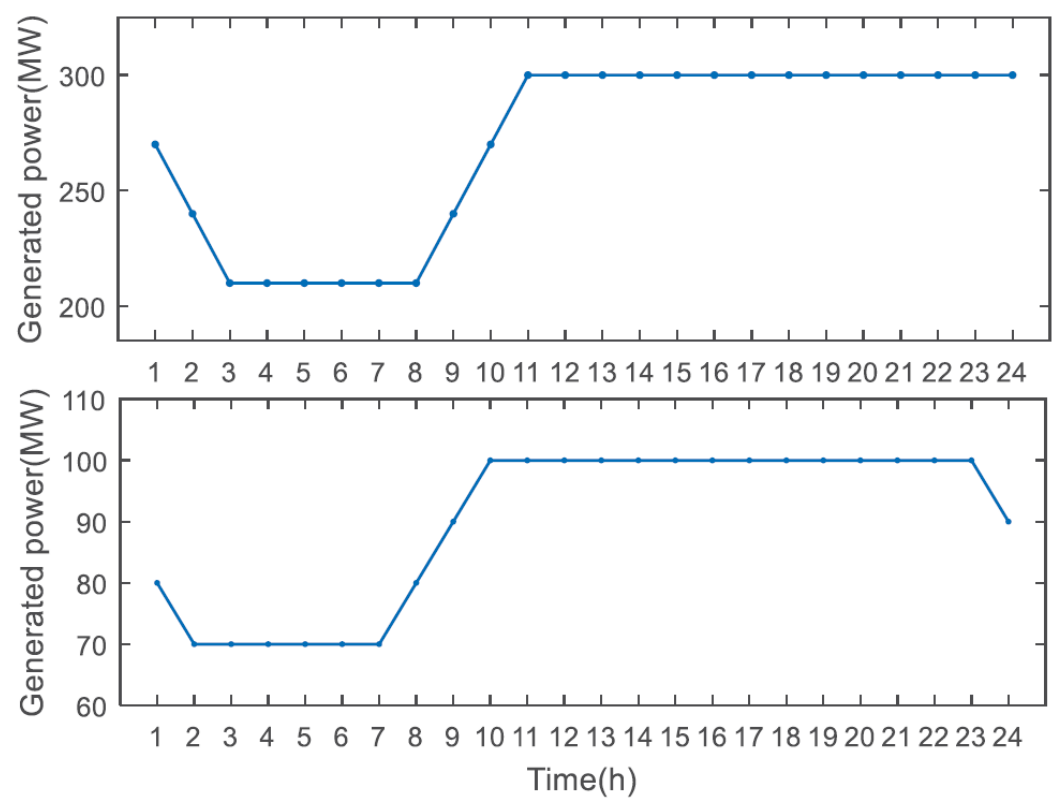

Figure 5. Injected active power of nuclear generation plants. 

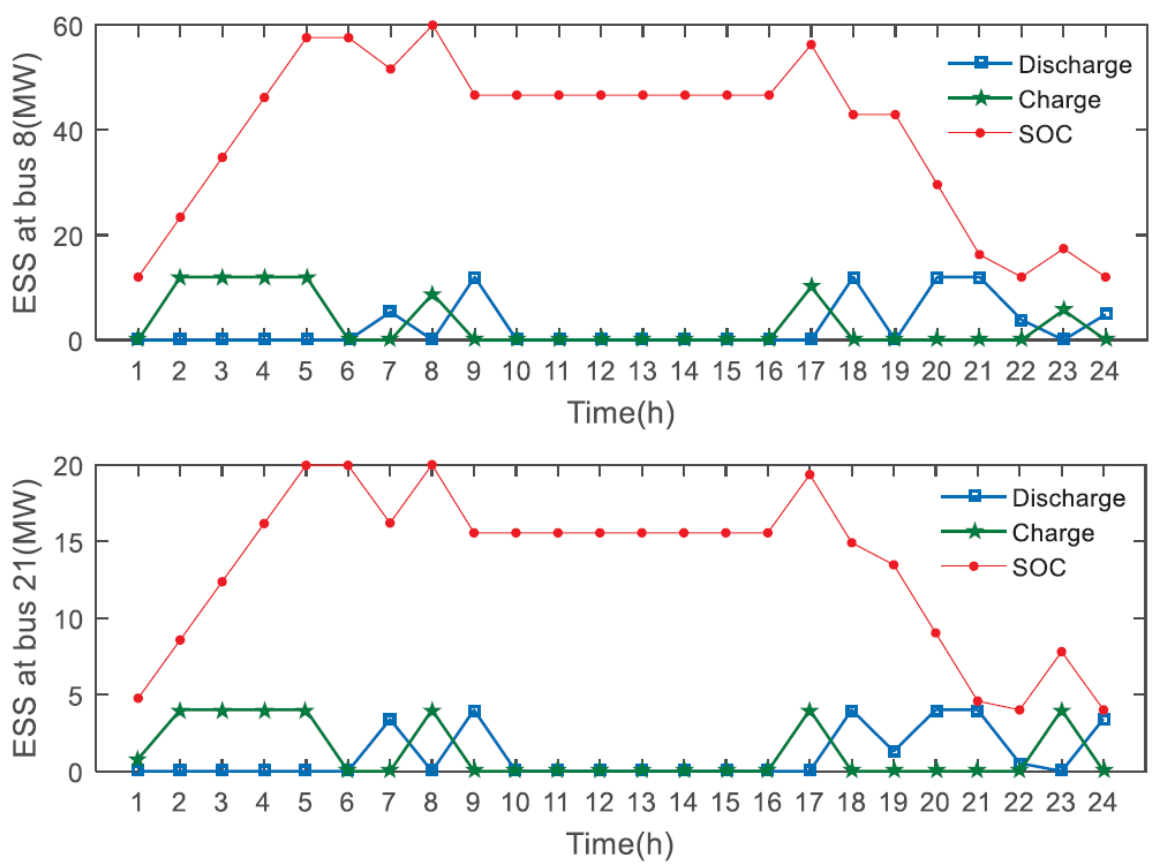

Figure 6. The hourly dispatch of the energy storage system (ESS) in a unit commitment (UC) problem.

\subsection{Energy Storage Parameters Impact on System Cost}

The ESS has both charging and discharging active power characteristics, which play an important role in peak-shaving.

For the UC model considering ESS, different energy storage parameters have different influences on the total cost. Table 3 illustrates that the overall system cost decreases when the active power capacity charging and discharging power limit increase.

Table 3. Total cost under different ESS parameters.

\begin{tabular}{cc}
\hline $\begin{array}{c}\text { ESS Parameters (Energy Storage Upper } \\
\text { Limit/MWh, Power Upper Limit/MW) }\end{array}$ & Total Cost/\$ \\
\hline 80,16 & $1.3337 \times 10^{6}$ \\
100,20 & $1.3336 \times 10^{6}$ \\
150,30 & $1.3264 \times 10^{6}$ \\
200,40 & $1.3239 \times 10^{6}$ \\
\hline
\end{tabular}

\subsection{Impact of Capacity Confidence Coefficients on System Cost}

Taking into account uncertainty when modelling reserve capacity gives more dexterity spare for power system scheduling.

To capture different degrees of net power fluctuation, different confidence levels are set. Figure 7 shows the hourly deterministic reserve capacity in comparison with the probabilistic reserve constraint at various confidence levels. Besides, as shown in Figure 8, the overall cost rises with confidence level increasing. 


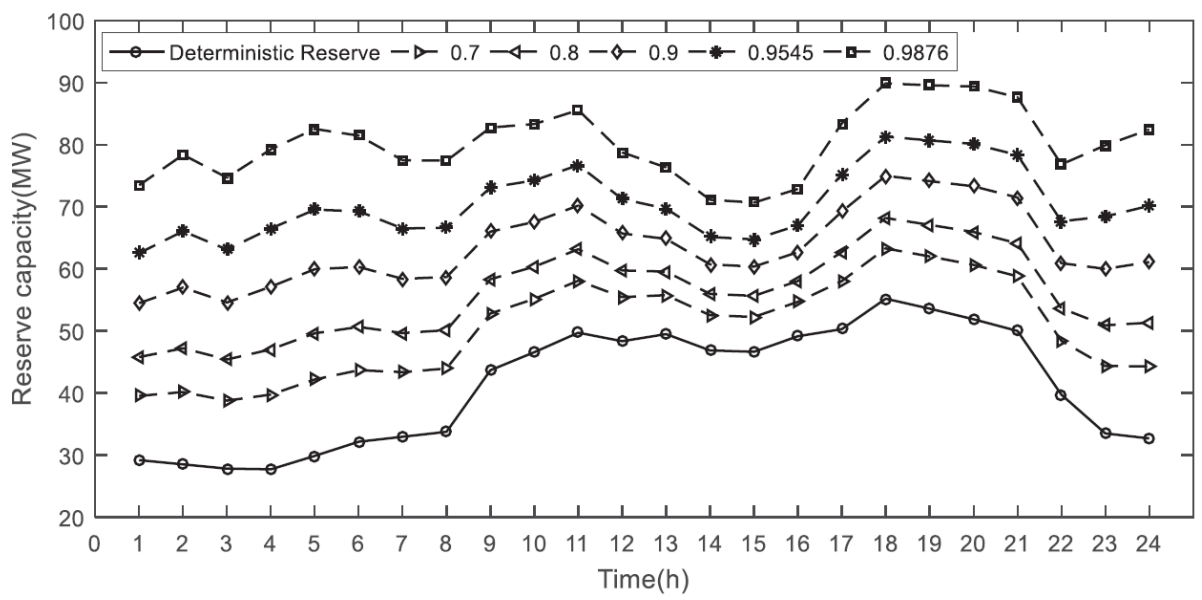

Figure 7. Reserve capacity under various confidence setups.

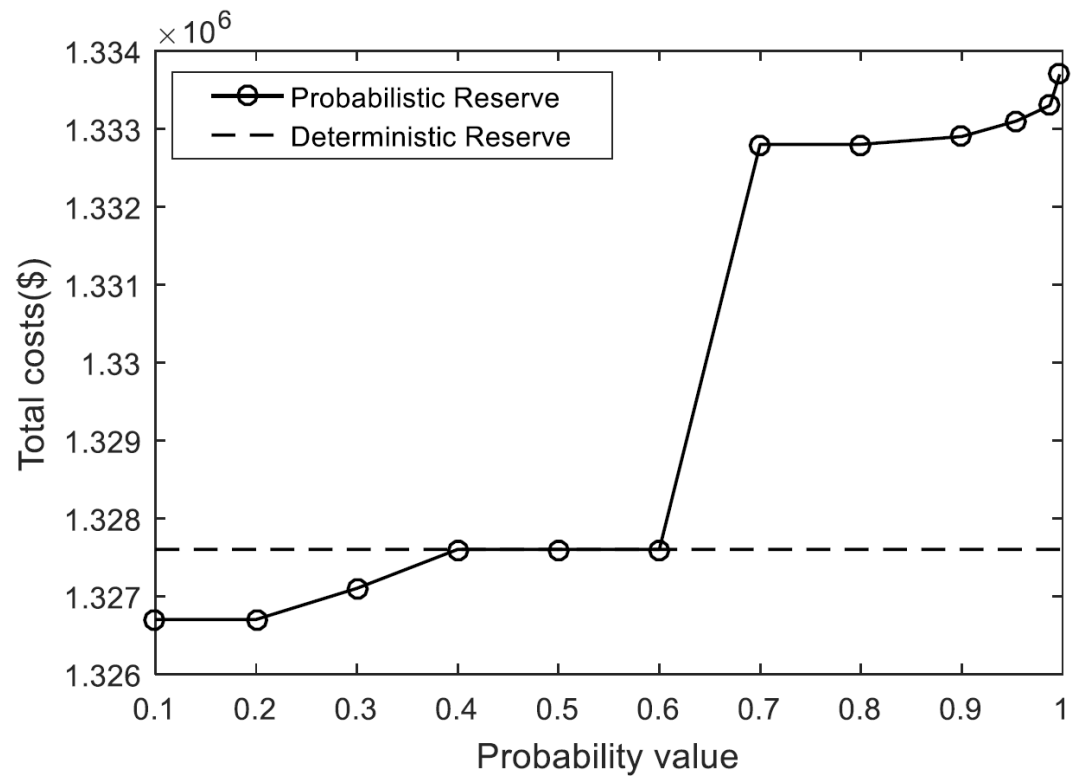

Figure 8. Total costs with different confidence level.

\section{Conclusions}

This paper presented a UC program that incorporated various generation types, such as thermal power, nuclear power, wind generation and ESS. The introduction of a nuclear power plant eases the peak-shaving pressure, decreases the up/down frequency of the conventional thermal power unit, and reduces the operational expenses. This ESS model makes scheduling more flexible, thus serving as an important adjunct function to generation system scheduling. This allows the grid to make full use of clean energy, such as solar and wind energy. In addition, a GMM has been applied to model wind power with non-gaussian uncertainty, and the spinning reserve setting of the system is more rational; this provides the dispatcher with more comprehensive choices. Regarding the influence of uncertainty on system scheduling, some alternatives have not yet been considered, and these will be studied in our future work.

Author Contributions: Conceptualization, Y.J. and J.W.; methodology, Y.J.; software, F.G.; validation, Y.J., J.W. and F.G.; formal analysis, Y.J.; investigation, J.W.; resources, Y.J.; data curation, Y.L.; writing-original draft preparation, Y.J.; writing - review and editing, Y.J. and J.W.; visualization, Y.L.; supervision, Y.J.; project administration, M.D.; funding acquisition, M.D., D.L., K.S. and H.Z. 
Funding: This paper is supported by Open Fund of FX83-18-0022018 year Beijing Key Laboratory of Demand Side Multi-Energy Carriers Optimization and Interaction Technique (No. YDB51201801084), the National Natural Science Foundation of China (Grant No. 51707196) and the State Key Laboratory of Alternate Electrical Power System with Renewable Energy Sources (Grant No. LAPS18015).

Acknowledgments: Yuntao Ju and Dezhi Li also belong to Beijing Key Laboratory of Demand Side Multi-Energy Carriers Optimization and Interaction Technique, Beijing 100192, China. Mingyu Dong and Kun Shi also belong to China Electric Power Research Institute, Haidian District, Beijing 100192, China.

Conflicts of Interest: The authors declare no conflict of interest.

\section{Abbreviations}

The following abbreviations are used in this manuscript:

UC unit commitment

ESS energy storage system

PWRs pressurized water reactors

GMM Gaussian mixture model

OF objective function

\section{Appendix A}

The power forecast error probability density function of wind farm represented by the GMM is expressed as:

$$
f^{(i)}\left(\Delta P_{W}^{(i)}\right)=\sum_{k=1}^{m} c_{k}^{(i)} g\left(u_{k}^{(i)}, \sigma_{k}^{(i)}, \Delta P_{W}^{(i)}\right)
$$

where, $m$ denotes the number of single Gaussian distributions in the GMM, and $c_{k}^{(i)}, u_{k}^{(i)}, \sigma_{k}^{(i)}$ denote the weight coefficient, mean and standard deviation, respectively, of the $k$ th single Gaussian distribution in wind farm $i$.

The total uncertain power of the system is expressed as:

$$
P_{\mathrm{un}}=\Delta p_{W 1}+\Delta p_{W 2}+\cdots+\Delta p_{W N}
$$

where, $N$ is the number of wind farms. Compared to the forecast error of wind power output power, the forecast error of load is small, so the forecast error of the load is ignored.

The change of active power flow of branch $s-t$ caused by the change of node active power output $\Delta p$ is $\Delta P_{s-t}$ in power system power flow analysis.

$$
\Delta P_{s-t, j}=G_{s-t, j} \Delta P_{W, j}
$$

where, $G_{s-t, j}$ is the distribution factor of generator output power transfer of node $j$ for line $s-t$. Considering that multiple nodes are connected to the wind farm, the influence of all wind turbines on a branch should be considered. The linear relationship between the power error of the line $s-t$ and all wind farm errors is expressed as:

$$
\Delta P_{S-t}=\beta^{(1)} \Delta p^{(1)}+\beta^{(2)} \Delta p^{(2)}+\cdots+\beta^{\left(N_{W}\right)} \Delta p^{\left(N_{W}\right)}
$$

where, $\beta^{(1)} \beta^{(2)} \ldots \beta^{\left(N_{W}\right)}$ are constant coefficients, which can be obtained by the wind turbine output power transfer distribution factor matrix $G$.

Several properties of probability calculation are introduced. Suppose that $x_{1}$ and $x_{2}$ are two independent random variables, and their probability density functions are $f^{\left(x_{1}\right)}(\cdot)$ and $f^{\left(x_{2}\right)}(\cdot)$, respectively. $y$ is the linear function of the random variables $x_{1}$ and $x_{2}$, and its probability density function is $f^{(y)}(\cdot)$.

(1) If $\mathrm{y}=h x_{1}$ and $h$ is a constant, then $f^{(\mathrm{y})}(y)=f^{\left(x_{1}\right)}(y / h) / h$.

(2) If $\mathrm{y}=x_{1}+x_{2}$, then $f^{(\mathrm{y})}(y)=f^{\left(x_{1}\right)}(y) \otimes f^{\left(x_{2}\right)}(y)$. 
(3) If $x_{1}$ and $x_{2}$ are subject to the normal distribution and $\mathrm{y}=x_{1}+x_{2}$, then $y$ is still subject to the normal distribution, the expected value of $y$ is the sum of the expected values of $x_{1}$ and $x_{2}$, and the variance of $y$ is the sum of the variance of $x_{1}$ and $x_{2}$.

According to the above properties, the probability density function of line power $P^{(s-t)}$ can be deduced as follows:

$$
f^{(s-t)}\left(p^{(s-t)}\right)=\sum_{k=1}^{3} \sum_{m=1}^{3} \ldots \sum_{n=1}^{3} c^{(s-t)} g\left(\bar{u}^{(s-t)}, \sigma^{(s-t)}, p^{(s-t)}\right)
$$

where

$$
\begin{gathered}
c^{(s-t)}=c_{k}^{(1)} c_{m}^{(2)} \cdots c_{n}^{\left(N_{w}\right)} \\
\bar{u}^{(s-t)}=\bar{p}^{s-t}+\beta_{s t}^{(1)} \bar{\mu}_{k}^{(1)}+\beta_{s t}^{(2)} \bar{\mu}_{k}^{(2)}+\cdots+\beta_{s t}^{\left(N_{w}\right)} \bar{\mu}_{k}^{\left(N_{w}\right)} \\
\left(\sigma^{(s-t)}\right)^{2}=\left(\beta_{s t}^{(1)} \sigma_{k}^{(1)}\right)^{2}+\left(\beta_{s t}^{(2)} \sigma_{m}^{(2)}\right)^{2}+\cdots+\left(\beta_{s t}^{\left(N_{w}\right)} \sigma_{n}^{\left(N_{w}\right)}\right)^{2}
\end{gathered}
$$

where, $\bar{p}^{s-t}$ is the expected value of the line power, which can be calculated by DC power flow. The cumulative distribution function of $\bar{p}^{s-t}$ is derived as follows:

$$
\begin{aligned}
& F^{(s-t)}\left(p^{(s-t)}\right)=\int_{-\infty}^{p^{(s-t)}} f^{(s-t)}(x) d x \\
& =\sum_{k=1}^{3} \sum_{m=1}^{3} \ldots \sum_{n=1}^{3} c^{(s-t)} \int_{-\infty}^{p^{(s-t)}} g\left(\bar{u}^{(s-t)}, \sigma^{(s-t)}, x\right) d x \\
& =\sum_{k=1}^{3} \sum_{m=1}^{3} \ldots \sum_{n=1}^{3} c^{(s-t)} \int_{-\infty}^{\frac{p^{(s-t)}-\bar{u}^{(s-t)}}{\sigma^{(s-t)}}} g(0,1, x) d x \\
& =\sum_{k=1}^{3} \sum_{m=1}^{3} \ldots \sum_{n=1}^{3} c^{(s-t)} G\left(\frac{p^{(s-t)}-\bar{u}^{(s-t)}}{\sigma^{(s-t)}}\right)
\end{aligned}
$$

where, $G(\cdot)$ denotes the cumulative distribution function of the standard Gaussian function.

The probability density function of random error variable $\Delta p^{(s-t)}$ can be deduced as follows:

$$
\begin{aligned}
& F^{(s-t)}\left(\Delta p^{(s-t)}\right)=\int_{-\infty}^{\Delta p^{(s-t)}} f^{(s-t)}(x) d x \\
& =\sum_{k=1}^{3} \sum_{m=1}^{3} \ldots \sum_{n=1}^{3} c^{(s-t)} \int_{-\infty}^{\Delta p^{(s-t)}} g\left(\bar{u}^{(s-t)}, \sigma^{(s-t)}, x\right) d x \\
& =\sum_{k=1}^{3} \sum_{m=1}^{3} \ldots \sum_{n=1}^{3} c^{(s-t)} \int_{-\infty}^{\frac{\Delta p^{(s-t)}-\mu^{(s-t)}}{\sigma^{(s-t)}}} g(0,1, x) d x \\
& =\sum_{k=1}^{3} \sum_{m=1}^{3} \ldots \sum_{n=1}^{3} c^{(s-t)} G\left(\frac{\Delta p^{(s-t)}-\mu^{(s-t)}}{\sigma^{(s-t)}}\right)
\end{aligned}
$$

where

$$
\begin{gathered}
c^{(s-t)}=c_{k}^{(1)} c_{m}^{(2)} \cdots c_{n}^{\left(N_{w}\right)} \\
u^{(s-t)}=\beta_{s t}^{(1)} \bar{\mu}_{k}^{(1)}+\beta_{s t}^{(2)} \bar{\mu}_{k}^{(2)}+\cdots+\beta_{s t}^{\left(N_{w}\right)} \bar{\mu}_{k}^{\left(N_{w}\right)} \\
\left(\sigma^{(s-t)}\right)^{2}=\left(\beta_{s t}^{(1)} \sigma_{k}^{(1)}\right)^{2}+\left(\beta_{s t}^{(2)} \sigma_{m}^{(2)}\right)^{2}+\cdots+\left(\beta_{s t}^{\left(N_{w}\right)} \sigma_{n}^{\left(N_{w}\right)}\right)^{2}
\end{gathered}
$$

Through formula (A9), sequence pairs of cumulative distribution functions of the system error and line power error variables are obtained:

$$
\begin{aligned}
& {\left[P_{u n 1}, F_{u n}\left(P_{u n 1}\right)\right],\left[P_{u n 2}, F_{u n}\left(P_{u n 2}\right)\right],\left[P_{u n 3}, F_{u n}\left(P_{u n 3}\right)\right] \cdots} \\
& {\left[\Delta p_{1}{ }^{(s-t)}, F^{(s-t)}\left(\Delta p_{1}{ }^{(s-t)}\right)\right],\left[\Delta p_{2}{ }^{(s-t)}, F^{(s-t)}\left(\Delta p_{2}{ }^{(s-t)}\right)\right],\left[\Delta p_{3}{ }^{(s-t)}, F^{(s-t)}\left(\Delta p_{3}{ }^{(s-t)}\right)\right] \cdots}
\end{aligned}
$$

where, the intervals of $P_{u n 1}, P_{u n 2}, P_{u n 3} \cdots$ and $\Delta p_{1}, \Delta p_{2}, \Delta p_{3} \cdots$ are homogeneous, so the inverse value of the cumulative distribution function corresponding to the specific probability value can be obtained 
by the sequence pairs query or the first order interpolation algorithm during the opportunity constraint optimization process.

\section{References}

1. Zhao, J.; Liu, D.; Lei, Q.; Du, Z.; Wang, J.; Zhou, L.; Huang, Y. Analysis of nuclear power plant participating in peak load regulation of power grid and combined operation with pumped storage power plant. Zhongguo Dianji Gongcheng Xuebao/Proc. Chin. Soc. Electr. Eng. 2011, 31, 1-6.

2. Fang, Y.; Chen, S.; Fang, Y. Research of Peaking Simulation on Nuclear Generating Units Based on Regulating Rod. Guangdong Electr. Power 2012, 25, 27-30.

3. Wu, P.; Yang, Y.; Chen, H.; Yi, J.; Bu, G. Research on operational characteristics and core simulation of the third-generation nuclear reactor AP1000. Dianwang Jishu/Power Syst. Technol. 2014, 38, 1196-1202.

4. Zhang, T.; Ma, X.D.; Zhu, Y.; Wang, G.; Ye, P. A Research Summary on Combined Peaking Load Strategies of Nuclear Power Plant. Adv. Mater. Res. 2014, 986-987, 196-201. [CrossRef]

5. Zhao, J.; Liu, D.; Yang, N.; Sun, W.; Wang, Q. Operation mode and benefits of nuclear power plant participating in peak load regulation of power system. Dianwang Jishu/Power Syst. Technol. 2012, 36, 250-255.

6. Wu, H.-Y.; Guan, X.-H.; Zhai, Q.-Z.; Gao, F. Short-term hydrothermal scheduling using mixed-integer linear programming. Proc. CSEE 2009, 29, 82-88.

7. Wang, J.; Zhao, J.; Ye, X.; Liu, D.; Weng, Y.; Wang, L.; Wang, H. Safety constraints and optimal operation of large-scale nuclear power plant participating in peak load regulation of power system. IET Gener. Transm. Distrib. 2017, 11, 3332-3340. [CrossRef]

8. Vatanpour, M.; Yazdankhah, A.S. The impact of energy storage modeling in coordination with wind farm and thermal units on security and reliability in a stochastic unit commitment. Energy 2018, 162, 476-490. [CrossRef]

9. Boyes, J.D.; Clark, N.H. Technologies for energy storage. Flywheels and super conducting magnetic energy storage. In Proceedings of the 2000 Power Engineering Society Summer Meeting (Cat. No.00CH37134), Seattle, WA, USA, 16-20 July 2000; Volume 3, pp. 1548-1550.

10. Skovajsa, J.; Zalesak, M. The Use of the Photovoltaic System in Combination With a Thermal Energy Storage for Heating and Thermoelectric Cooling. Appl. Sci. 2018, 8, 1750. [CrossRef]

11. Zanabria, C.; Andrén, F.P.; Kathan, J.; Strasser, T.I. Rapid Prototyping of Multi-Functional Battery Energy Storage System Applications. Appl. Sci. 2018, 8, 1326. [CrossRef]

12. Soroudi, A.; Siano, P.; Keane, A. Optimal DR and ESS Scheduling for Distribution Losses Payments Minimization Under Electricity Price Uncertainty. IEEE Trans. Smart Grid 2015, 7, 261-272. [CrossRef]

13. Zheng, L.; Hu, W.; Lu, Q.; Min, Y.; Yuan, F.; Gao, Z. Research on planning and operation model for energy storage system to optimize wind power integration. Zhongguo Dianji Gongcheng Xuebao/Proc. Chin. Soc. Electr. Eng. 2014, 34, 2533-2543.

14. Wang, Y.; Xia, Q.; Kang, C. Unit Commitment With Volatile Node Injections by Using Interval Optimization. IEEE Trans. Power Syst. 2011, 26, 1705-1713. [CrossRef]

15. Wang, Y.; Zhou, G.; Han, H.; Li, T.; Ding, Q.; Zhang, K. Unit commitment considering optimal confidence of wind power uncertainty. Dianwang Jishu/Power Syst. Technol. 2017, 41, 808-815.

16. Ai, X.; Tayierjiang, B.; Yang, L.; Yang, J.; Fang, J.; Wen, J. Optimizing the Spinning Reserve in Wind Power System Using Scenario Method. Dianwang Jishu/Power Syst. Technol. 2018, 42, 835-841.

17. Liu, B.; Feng, L.; Wang, C.; Mei, S.; Wei, W. Unit commitment considering flexibility and uncertainty of wind power generation. Dianwang Jishu/Power Syst. Technol. 2015, 39, 730-736.

18. Gao, H.; Liu, J.; Wei, Z.; Cao, Y.; Wang, W.; Huang, S. A security-constrainted dispatching model for wind generation units based on extreme scenario set optimization. Dianwang Jishu/Power Syst. Technol. 2013, 37, 1590-1595.

19. Singh, R.; Pal, B.C.; Jabr, R.A. Distribution system state estimation through Gaussian mixture model of the load as pseudo-measurement. IET Gener. Transm. Distrib. 2010, 4, 50-59. [CrossRef]

20. Doherty, R.; O'Malley, M. A New Approach to Quantify Reserve Demand in Systems with Significant Installed Wind Capacity. IEEE Trans. Power Syst. 2005, 20, 587-595. [CrossRef] 
21. Ortega-Vazquez, M.; Kirschen, D.S. Estimating the Spinning Reserve Requirements in Systems with Significant Wind Power Generation Penetration. IEEE Trans. Power Syst. 2009, 24, 114-124. [CrossRef]

22. Arroyo, J.; Conejo, A.J. Optimal Response of a Thermal Unit to an Electricity Spot Market. IEEE Trans. Power Syst. 2000, 15, 1098-1104. [CrossRef]

(C) 2019 by the authors. Licensee MDPI, Basel, Switzerland. This article is an open access article distributed under the terms and conditions of the Creative Commons Attribution (CC BY) license (http:/ / creativecommons.org/licenses/by/4.0/). 